\title{
Auton'Al 60: Prevention program for the loss of autonomy of elderly in France
}

\author{
Laura Lavefve, Julie Branchu, Béatrice Breil, Flore Depeint, Philippe Pouillart, \\ Claude-Narcisse Niamba, Hassan Younes, Cécile Buche-Foissy and Anne-Kathrin Illner \\ Polytechnic Institute UniLaSalle, College of Health Sciences, Beauvais, France
}

\section{Abstract}

Introduction: The loss of autonomy in elderly varies according to the individual health status, but also to the social and psychological environment. Multiple risk factors play a role, including limited access to a balanced diet, physical inactivity or a poor social network. Prevention programs must therefore be based on a multidimensional approach, but are rarely studied with regard to their effectiveness.

Materials and methods: The objective of the Auton'Al 60 program is to prevent the loss of autonomy related to diet, physical activity and mental health in elderly, aged 60 to 89, in the county of Oise, North of France. Between February and July 2018 , 7 different prevention workshops were conducted in 5 geographical areas (urban and rural). A blog and a monthly newsletter have been created to limit the loss to follow- up. Evaluation was based on an in-depth interview at T0 inquiring about the level of autonomy, the dietary habits and the health status, as well as on 2 self-administered questionnaires during and at the end of the program. In addition, each workshop has been evaluated separately according to key indicators. Statistical analysis was performed using SPSS 17.

Results: 69 workshops have been completed during 5 months. In total, 91 subjects $(27.8 \%$ men - $71.9 \%$ women) participated in at least one workshop, with a mean age of 69.1 years $( \pm 6.3) .72 .6 \%$ of the subjects were present at 6 or 7 workshops, and $49.5 \%$ participated in all activities. A state of loss of autonomy was reported by $6.7 \%$ of the participants. $32.6 \%$ of the population were chronic-disease-free and $43.8 \%$ had a normal BMI $\left(\mathrm{kg} / \mathrm{m}^{2}\right)$. The prevalence of obesity was $15.7 \%$. The program showed effectiveness to decrease the consumption of high-fat products in $52.9 \%$ as well as the consumption of sugary products in $60.9 \%$ of the participants. Furthermore, $23.2 \%$ of the subjects started a new social activity during the program. However, compared to other age groups observed fragility seemed to be particularly high in elderly aged from 66 to 69 years.

Conclusion: The Auton'Al 60 program confirms the heterogeneity in the level of autonomy, health status and health behavior in elderly. The multidimensional approach has shown satisfactory effectiveness. Prevention strategies have been developed for isolated or low-income subjects and are under current evaluation.

\section{Conflict of Interest}

There is no conflict of interest. Ms C. BUCHE-FOISSY and Dr AK ILLNER have a joint senior authorship. 